\title{
Interview with Yufei Zhao
}

\section{Toufik Mansour}

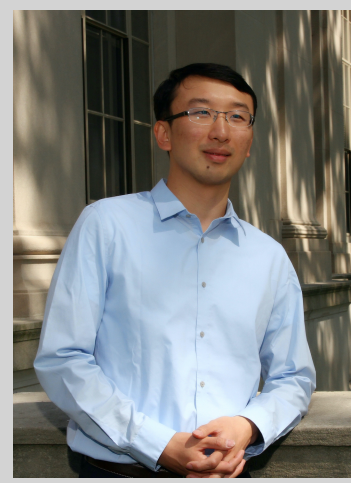

Yufei Zhao received his Ph.D. in mathematics from the Massachusetts Institute of Technology (MIT) under the supervision of Jacob Fox. He is currently Assistant Professor of Mathematics at MIT. He has held postdoctoral and visiting positions at Stanford University, UC Berkeley and Oxford. Professor Yufei Zhao made significant contributions in combinatorics. His major contribution is related to the celebrated Green-Tao theorem. Zhao's proof, co-authored with Jacob Fox and David Conlon, simplifies a central part of the proof, allowing a more direct approach to the Green-Tao theorem. Their work improves the understanding of pseudorandom structures and has other applications in mathematics and computer science. For his contributions, Professor Zhao has received several awards including the Dénes König Prize (2018) and a Sloan Research Fellowship (2019).

Mansour: Professor Zhao, first of all, we would like to thank you for accepting this interview. Would you tell us broadly what combinatorics is?

Zhao: It is an honor to be interviewed by ECA. Combinatorics concerns the study of discrete structures such as graphs, set systems, and geometric configurations. Many problems in combinatorics can be simply stated requiring minimal background to understand, but the solutions sometimes use powerful techniques and/or ingenious insights. Modern combinatorics often draws ideas from other mathematical areas, for example, probabilistic, algebraic, Fourier analytic, topological, and geometric methods.

Mansour: What do you think about the development of the relations between combinatorics and the rest of mathematics?

Zhao: I am drawn to combinatorics problems that have rich connections to other areas of mathematics. A great example is Szemerédi's theorem ${ }^{1}$. While Szemerédi initially proved his result combinatorially, subsequently other proofs were found using completely different methods, most notably via (1) ergodic theory, (2) higher order Fourier analysis, and (3) hypergraph regularity. Each approach involves sophisticated ideas and has led to its own exciting direction of research. Together, they reveal deep structures in Szemerédi's theorem, showing that what we have is not merely some isolated combinatorial curiosity, but rather it is integrally tied to the rest of mathematics. Terry Tao uses Szemerédi's theorem as the core example in this excellent essay What is good mathematics? ${ }^{2}$.

Mansour: What have been some of the main goals of your research?

Zhao: I am interested in solving extremal problems in combinatorics - what is the maximum or minimum size of a structure with given properties?

Much of my Ph.D. work concerns building graph theoretic techniques that connect to additive combinatorics, thereby further bridging the two subjects. I am continuing to work in this direction.

The authors: Released under the CC BY-ND license (International 4.0), Published: January 1, 2021

Toufik Mansour is a professor of mathematics at the University of Haifa, Israel. His email address is tmansour@univ.haifa.ac.il

${ }^{1}$ E. Szemerédi, On sets of integers containing no $k$ elements in arithmetic progression, Acta Arith. 27 (1975), $199-245$.

${ }^{2}$ T. Tao, What is good mathematics?, Bull. Amer. Math. Soc. (N.S.) 44 (2007), 623-634. 
Lately I have been working on extremal problems in discrete geometry, for example, equiangular lines and the joints problem. One good problem often leads to another. For example, studying equiangular lines led us to a new result in spectral graph theory: a connected bounded degree graph has sublinear second eigenvalue multiplicity ${ }^{3}$.

Mansour: We would like to ask you about your formative years. What were your early experiences with mathematics? Did that happen under the influence of your family or some other people?

Zhao: I have always liked math and numbers from as young as I can remember. I grew up in China and then moved to Canada when I was eleven. In China, the school environment was encouraging for someone who was good at math. When we moved to Canada, I initially had a hard time adjusting due to language and cultural barriers. It frustrated me that no one at my school cared about mathematics. Fortunately I brought a few math books with me, and I spent a lot of time reading them. Mathematics was my sanctuary.

No one in my family is anywhere close to being a mathematician, so it was a bit surprising to them that I ended up becoming one. When I grew up in China, we lived with my paternal grandfather, who was a middle school Chinese teacher, and his educational philosophy was that kids need to learn to sit still for a long time and concentrate. That turned out to be a good skill to have for doing mathematics!

Mansour: Were there specific problems that made you first interested in combinatorics?

Zhao: As an undergraduate, I took a variety of classes across many areas of mathematics. Combinatorics is the subject that resonated with me the most. I enjoyed the problem solving nature of combinatorics. There weren't any specific problems that got me interested in combinatorics. As an undergraduate, I enjoyed taking several classes from Prof. Richard Stanley in enumerative and algebraic combinatorics, though what I do now is quite different.

Between undergraduate studies and Ph.D., I spent a year at Cambridge University as a
Gates Scholar studying Part III of the Mathematical Tripos. I learned a lot from taking combinatorics classes from Imre Leader, David Conlon, and Ben Green. I became really interested in extremal combinatorics, and I was ready to start doing research when I got back to MIT.

Mansour: What was the reason you chose MIT for your Ph.D. and your advisor, Jacob Fox?

Zhao: I had several nice options for graduate programs in the US, but at the time, very few had a strong combinatorics presence (the situation has improved a lot since then). When I finished undergraduate, I talked to Jacob Fox, who was very encouraging. He recommended me to read the textbook The Probabilistic Method by Alon and Spencer. I really liked the book and had a fun time working through the exercises. When I got back to MIT from Cambridge to start my Ph.D., Fox suggested to me a long list of recent research papers to read. We discussed these papers regularly and intensely, and my research progressed very quickly from there.

Mansour: What was the problem you worked on in your thesis?

Zhao: The main problem that I worked on for my thesis is understanding extensions of Szemerédi's regularity lemma to sparse pseudorandom graphs. Szemerédi's regularity lemma ${ }^{4}$ is a power tool in combinatorics. But the original form of the method does not immediately say anything useful unless the graph has quadratically many edges. There was a lot of interest in extending the regularity method to sparser settings.

Most interesting applications of the regularity method have two separate components: (1) obtaining a regular partition of the vertex set and (2) proving a counting/embedding lemma to find desired structures among the regular parts. It was already known how to extend the first step to sparser graphs, but the second step posed significant difficulty. My first paper $^{5}$ in graduate school (coauthored with David Conlon and Jacob Fox) developed a counting lemma for regular partitions of sparse pseudorandom graphs. This already had in-

\footnotetext{
${ }^{3}$ Z. Jiang, J. Tidor, Y. Yao, S. Zhang and Y. Zhao, Equiangular lines with a fixed angle, arXiv:1907.12466.

${ }^{4}$ E. Szemerédi, Regular partitions of graphs, Problèmes combinatoires et théorie des graphes (Colloq. Internat. CNRS Univ. Orsay, Orsay, 1976), vol. 260, CNRS, 1978, pp. 399-401.

${ }^{5}$ D. Conlon, J. Fox and Y. Zhao, Extremal results in sparse pseudorandom graphs, Adv. Math. 256 (2014), 206-290.
} 
teresting applications. Then we took one step further and extended the techniques for hypergraphs $^{6}$. It turned out that our new method gave a simplified proof of the Green-Tao theorem on arithmetic progressions in the primes.

I spent a lot of time in my Ph.D. thinking about the connections between graph theory and additive combinatorics. When I first started teaching as faculty, I designed and taught a course that combined these two areas together and highlighted their common themes. My goal was to teach the background to my research so that interested students could also start working in this direction (and this worked!). I built a nice set of lecture notes, produced with the help of my students, and I am working on turning these notes into a textbook. Also, all my lectures in Fall 2019 were videotaped and produced by MIT OpenCourseWare, freely available online to anyone who is interested ${ }^{7}$.

Mansour: What would guide you in your research? A general theoretical question or a specific problem?

Zhao: When I'm starting in a new direction, I like to have a few concrete problems in mind. Recently I have been thinking a lot about extremal problems in discrete geometry, and usually there is some bound that we are trying to improve. For example, for equiangular lines, the natural problem is to determine the maximum number of lines in $\mathbb{R}^{d}$ pairwise forming the same angle.

Once I have thought enough about an area, I would develop a sense of what general directions should be further investigated. Sparse regularity is a good example - the problems here are often less well specified. I look for the right questions to ask and think about what is missing in our understanding of the subject.

Mansour: When you are working on a problem, do you feel that something is true even before you have the proof?

Zhao: Usually, but sometimes there are surprises. I find it useful to make "micro- conjectures" while working on a problem, especially when working with collaborators. These micro-conjectures are concrete and falsifiable statements that act as guide posts. While micro-conjectures are usually not significant enough to merit publication on their own, resolving them nevertheless provides me with a sense of progress and accomplishment.

Mansour: What three results do you consider the most influential in combinatorics during the last thirty years?

Zhao: Here are my three (slightly biased by my own interests, of course), listed in no particular order. I chose these developments not just because of the longstanding problems that each solved, but also because the ideas introduced by the solutions led to paradigm shifts in the field. Each work is followed by a lot of other nice works, and the tools introduced have become central in the field - it would be foolish to not at least try the new tools when working on a related problem.

(1) Gowers' new proof of Szemerédi's theorem introduced higher order Fourier analysis ${ }^{8}$. This is a deep extension of the Fourier analytic proof of Roth's theorem. Gowers' ideas led to a significant portion of subsequent research in additive combinatorics, for example, the Green-Tao theorem ${ }^{9}$, and the Green-TaoZiegler inverse theorem for the Gowers uniformity norm ${ }^{10}$.

(2) Dvir's stunningly short proof of the finite field Kakeya conjecture brought new light to the powerful polynomial method in combinatorics ${ }^{11}$. Guth and Katz further developed Dvir's ideas in incidence geometry by applying the polynomial method to solve the joints problem ${ }^{12}$ and the Erdős distinct distances problem ${ }^{13}$. Guth also made advances on the restriction problems in harmonic analysis using these ideas ${ }^{14,15}$, which also led to improvements on the Kakeya problem. The polynomial method, and in particular, the polynomial partitioning technique, is now an indispensable tool for such problems. I recommend

\footnotetext{
${ }^{6}$ D. Conlon, J. Fox and Y. Zhao, A relative Szemerédi theorem, Geom. Funct. Anal. 25 (2015), 733-762.

${ }^{7}$ Y. Zhao, Graph theory and additive combinatorics, MIT OpenCourseWare, 2019, https://ocw.mit.edu/18-217F19.

${ }^{8}$ W. T. Gowers, A new proof of Szemerédi's theorem, Geom. Funct. Anal. 11 (2001), 465-588.

${ }^{9} \mathrm{~B}$. Green and T. Tao, The primes contain arbitrarily long arithmetic progressions, Ann. of Math. (2) 167 (2008), $481-547$.

${ }^{10}$ B. Green, T. Tao and T. Ziegler, An inverse theorem for the Gowers $U^{s+1}[N]-n o r m$, Ann. of Math. 176 (2012), $1231-1372$.

${ }^{11}$ Z. Dvir, On the size of Kakeya sets in finite fields, J. Amer. Math. Soc. 22 (2009), 1093-1097.

${ }^{12}$ L. Guth and N. H. Katz, Algebraic methods in discrete analogs of the Kakeya problem, Adv. Math. 225 (2010), $2828-2839$.

${ }^{13}$ L. Guth and N. H. Katz, On the Erdös distinct distances problem in the plane, Ann. of Math. (2) 181 (2015), $155-190$.

${ }^{14} \mathrm{~L}$. Guth, A restriction estimate using polynomial partitioning, J. Amer. Math. Soc. 29 (2016), 371-413.

${ }^{15}$ L. Guth, Restriction estimates using polynomial partitioning II, Acta Math. 221 (2018), 81-142.
} 
Guth's excellent textbook Polynomial methods in combinatorics for an introduction.

(3) The hypergraph container theorem, proved by Balogh, Morris, and Samotij ${ }^{16}$, and independently Saxton and Thomason ${ }^{17}$, gives us powerful tools to count independent sets in hypergraphs. The container method has lots of powerful applications in combinatorics, since many problems can be recast in terms of independent sets. These developments built on earlier graph container ideas of Kleitman and Winston $^{18}$ and Sapozhenko ${ }^{19}$.

Mansour: What are the top three open questions in your list?

Zhao: This is a hard one since I keep on getting interested in new problems as I learn more.

(1) Maximum sphere packing density in high dimensions

(2) Sidorenko's conjecture on graph homomorphism densities

(3) Shannon capacity of odd cycles

Mansour: What kind of mathematics would you like to see in the next ten-to-twenty years as the continuation of your work?

Zhao: I would like to see more analytic and algebraic methods applied to solve extremal combinatorics problems. And more broadly, I would like to see methods and ideas from other areas of mathematics applied to solve combinatorics problems.

My analytic perspective towards combinatorics was influenced by papers of Gowers, Lovász, Tao, and others that I read during my Ph.D. They favored viewing discrete structures such as graphs and hypergraphs analytically in terms of functions. I have personally found this perspective both instructive and useful in my own work. Perhaps a unified perspective might be distilled into some sort of "analytic graph theory" similar to analytic number theory.

Algebraic methods are magical. Once in a while, some longstanding conjecture gets cracked in an ingenious one-page algebraic "book proof." Some notable recent examples include the finite field Kakeya problem ${ }^{11}$, the cap set problem ${ }^{20,21}$, and the sensitivity con- jecture $^{22}$. Why do algebraic methods process such stunning magic? And where will we find the next gem?

Mansour: Do you think that there are core or mainstream areas in mathematics? Are some topics more important than others?

Zhao: One should be careful when comparing different areas of mathematics. Every selfrespecting mathematician will tell you why the problems they are working on are interesting and important. They probably have some good reasons, but it is really hard to tell in the moment which directions will become most important in the future.

A great example is the Erdős distinct distances problem, which asks to determine the smallest number of pairwise distances determined by $N$ points on a plane. This problem may seem like a curious puzzle, but its eventual solution by Guth and Katz ${ }^{13}$ led to the powerful method of polynomial partitioning, which is now a central tool not only in combinatorial geometry but also for the restriction problem in harmonic analysis. As such, the distinct distances problem is a great problem, but had we not seen the solution, it would have been hard to say exactly why it is so great.

Mansour: What do you think about the distinction between pure and applied mathematics that some people focus on? Is it meaningful at all in your case? How do you see the relationship between so-called "pure" and "applied" mathematics?

Zhao: I do not think it is too productive to focus on this distinction. To me the main difference comes from the source and motivation of the investigation - does it mostly come from within mathematics or from outside mathematics?

Some of my work, for example, on property testing, overlaps with theoretical computer science. One can tell a nice story about how this mathematics could be applied to check properties of large objects. Though, when actually working on the problem, we are primarily focused on trying to prove a nice theorem.

\footnotetext{
${ }^{16}$ J. Balogh, R. Morris and W. Samotij, Independent sets in hypergraphs, J. Amer. Math. Soc. 28 (2015), 669-709.

${ }^{17}$ D. Saxton and A. Thomason, Hypergraph containers, Invent. Math. 201 (2015), 925-992.

${ }^{18}$ D. J. Kleitman and K. J. Winston, On the number of graphs without 4-cycles, Discrete Math. 41 (1982), $167-172$.

${ }^{19}$ A. A. Sapozhenko, On the number of independent sets in extenders, Diskret. Mat. 13 (2001), 56-62.

${ }^{20}$ E. Croot, V. F. Lev and P. P. Pach, Progression-free sets in $\mathbb{Z}_{4}^{n}$ are exponentially small, Ann. of Math. (2) 185 (2017), 331-337.

${ }^{21}$ J. S. Ellenberg and D. Gijswijt, On large subsets of $\mathbb{F}_{q}^{n}$ with no three-term arithmetic progression, Ann. of Math. (2) 185 (2017), 339-343.

${ }^{22} \mathrm{H}$. Huang, Induced subgraphs of hypercubes and a proof of the sensitivity conjecture, Ann. of Math. (2) 190 (2019), 949-955.
} 
Mansour: You have extensive experience with major mathematics competitions such as International Mathematics Olympiads and Putnam. How much do you think that mathematics competitions inspire young students for a research career?

Zhao: I credit a lot of my own early development in mathematics to math competitions. I found them really fun. The competitions motivated me to learn more mathematics. They were also a great way to test my understanding - you can't just talk the talk. In competition training, there is a rapid feedback loop, which is an effective way to learn skills such as proof-writing.

I also really enjoyed meeting other kids in math camps and competitions. It provided me with a community that encouraged me to keep working hard to improve my mathematics. Some people I met through math competitions remain my closest friends.

In terms of transitioning from math competitions to math research, I like the analogy of comparing sprints to marathons. They are not the same sport. Being good at one is neither necessary nor sufficient for being good at the other. But they are not completely uncorrelated either. If you are a good sprinter, you are likely in good physical shape, and that helps with running long distance, though there are of course also many other important factors.

I find that students with extensive math competition training tend to have strong foundations and technical skills. They also generally like tackling challenging problems. To transition into a successful research career, they need to pick up many other skills, for example, choosing good problems to work on, and persistence. A mentor once emphasized to me the utmost importance of developing good mathematical taste. A little guidance and encouragement here can go a long way.

Mansour: What advice would you give to young people thinking about pursuing a research career in mathematics?

Zhao: It takes a lot of work, and sometimes a little bit of luck. You have to be focused and dedicated. Find a problem that you cannot stop thinking about. Obsession is the key. Enjoy the process. Talk to people. Learn from them. Work with them. Always keep on learning something new.
Mansour: Would you tell us about your interests besides mathematics?

Zhao: I like exploring new technology and playing with new software and tech gadgets. Sometimes they improve my productivity, but sometimes it is just for fun, and making sure that I am not falling behind the times.

I love to swim for fun (never competitively). Before the COVID-19 epidemic, I would swim in the university pool almost every day. When I swim, sometimes I think about a math problem, but mostly I just use it to clear my mind. It is like a moving meditation.

Mansour: Before we close this interview with one of the young promising leaders in combinatorics, we would like to ask some more specific mathematical questions. One of your main contributions is closely related to the GreenTao theorem. Would you tell us about the Green-Tao theorem and your related work?

Zhao: The Green-Tao theorem ${ }^{9}$ says that the primes contain arbitrarily long arithmetic progressions. I actually remember first hearing about this result in high school. I was stunned by the elegance of this statement, even though at the time the proof was too advanced for my understanding.

In my Ph.D., my work on the sparse regularity method naturally led me to the Green-Tao theorem once again.

Szemerédi's theorem ${ }^{1}$ says that every subset of integers with positive density contains arbitrarily long arithmetic progressions. This is a deep theorem, and as I mentioned earlier, while we now know several proofs of Szemerédi's theorem, none of them can be considered easy.

On the other hand, the asymptotic density of the primes is zero, and Szemerédi's theorem does not directly apply to the primes. Nevertheless, Green and Tao devised a powerful "transference principle" to adapt Szemerédi's theorem as a black box from the dense setting to the sparse setting.

They set up some sparse set of integers, called the "almost primes" (integers without small prime divisors), and showed that the primes sit inside as a relatively dense subset. These almost primes furthermore have some nice pseudorandomness properties.

Green and Tao then proved a relative Szemerédi theorem, showing that every set of integers satisfying certain pseudorandomness hy- 
potheses must also satisfy a relativized Szemerédi's theorem, namely that every relatively dense subset contains long arithmetic progressions.

With Conlon and Fox ${ }^{6}$, we proved a new and much simpler relative Szemerédi theorem that require much weaker pseudorandomness hypotheses compared to the original GreenTao work. The new pseudorandomness hypotheses are quite natural. They can be viewed as a graphical analog of having small second moments.

We wrote an exposition of the GreenTao theorem ${ }^{23}$, which includes a self-contained proof (other than Szemerédi's theorem, which is applied as a black box). Our exposition incorporates all the simplifications to the proof to date, and emphasizes a graph theoretic perspective.

Mansour: In a very recent paper, you and your co-authors, have introduced and developed a theory of limits for sequences of sparse graphs based on $L^{p}$ graphons. These results generalize both the existing $L^{\infty}$ theory of dense graph limits and its extension by Bollobás and Riordan to sparse graphs without dense spots. Would you tell us about this work and possible future research?

Zhao: Lovász developed an influential theory of graph limits ${ }^{24}$. The classical graph limit theory is applicable to graphs with constant edge density. It remains an interesting challenge, from both theory and applications, to further develop a sparse theory of graph limits. I was naturally drawn to this problem of extending graph limit theory to sparse settings, since it is related to sparse regularity.

I spent time at Microsoft Research during my Ph.D. and worked with Christian Borgs, Jennifer Chayes, and Henry Cohn, and we developed a notion of graph convergence and limits for certain families of sparse graphs ${ }^{25,26}$. One of our motivations was to devise a frame- work for studying graphs with power law degree distributions, which are very popular in network theory due to their observed prevalence in nature and reality. These networks have heavy tail degree distributions, and they do not fall in the existing frameworks of sparse graph limit theories. Our $L^{p}$ theory of graph convergence and graph limits turned out to be a mathematically elegant framework that also includes power law degree distributions.

I am still quite interested in mathematical problems on this topic. Bollobás and Riordan, in their seminal paper on sparse graph $\operatorname{limits}^{27}$, formulated a long list of conjectures, which would all be desirable properties of a nice general sparse graph limit theory. Recently, together with my students Ashwin Sah, Mehtaab Sawhney, and Jonathan Tidor ${ }^{28}$, we found a single counterexample that refutes every conjecture in Bollobás and Riordan's paper. This example shows that sparse graphs can behave much more wildly than previously expected, so it actually opens doors to a lot more exciting possibilities!

Mansour: In your paper "The number of independent sets in a regular graph" published in 2010, you used a short and elegant counting argument to reduce the general case to a particular case, the bipartite case. Would you tell us about this result and the important ideas behind the main result? In general, how do enumerative techniques play a role in your research?

Zhao: I worked on this problem as an undergraduate during a summer research program at Duluth run by Joe Gallian. I was initially working on an additive combinatorics problem suggested to me by Gallian, and in doing so I needed to show that certain 4-regular graphs must not have too many independent sets. I looked up the literature on this topic, and found a paper by Jeff Kahn that proved a tight bound for bipartite graphs ${ }^{29}$. I actu-

\footnotetext{
${ }^{23}$ D. Conlon, J. Fox and Y. Zhao, The Green-Tao theorem: an exposition, EMS Surv. Math. Sci. 1 (2014), $249-282$.

${ }^{24}$ L. Lovász, Large networks and graph limits, American Mathematical Society Colloquium Publications, vol. 60, American Mathematical Society, Providence, RI, 2012.

${ }^{25} \mathrm{C}$. Borgs, J. T. Chayes, H. Cohn and Y. Zhao, An $L^{p}$ theory of sparse graph convergence I: Limits, sparse random graph models, and power law distributions, Trans. Amer. Math. Soc. 372 (2019), 3019-3062.

${ }^{26} \mathrm{C}$. Borgs, J. T. Chayes, H. Cohn and Y. Zhao, An L $L^{p}$ theory of sparse graph convergence II: LD convergence, quotients and right convergence, Ann. Probab. 46 (2018), 337-396.

${ }^{27}$ B. Bollobás and O. Riordan, Metrics for sparse graphs, Surveys in combinatorics, vol. 365, Cambridge Univ. Press, Cambridge, 2009, pp. 211-287.

${ }^{28}$ A. Sah, M. Sawhney, J. Tidor and Y. Zhao, A counterexample to the Bollobás-Riordan conjectures on sparse graph limits, Combin. Probab. Comput., to appear.

${ }^{29}$ J. Kahn, An entropy approach to the hard-core model on bipartite graphs, Combin. Probab. Comput. 10 (2001), $219-237$.
} 
ally needed the bound for non-bipartite graphs, which Kahn left open as a conjecture in his paper, and this is what I solved ${ }^{30}$.

The independent set in regular graphs problem asks to determine the maximum possible number of independent sets in a $d$-regular $n$ vertex graph. A better way to state the problem is to allow $n$ to vary: maximize $i(G)^{1 / v(G)}$ among $d$-regular graphs $G$ for a given $d$. The problem turned out to be much more interesting than the original additive combinatorics problem that I started with.

This independent set problem was first raised by Granville in the 1980's in connection with the Cameron-Erdös conjecture on the number of sum-free sets. Alon ${ }^{31}$ proved the first non-trivial bound, and conjectured that the maximizing $G$ should be a disjoint union of $K_{d, d}$ 's. Kahn independently came to the problem from studying the hard-core model in statistical physics, and using a clever entropy argument, he proved the conjecture in the case of bipartite graph $G$, and also conjectured that the bipartite hypothesis can be removed.

I was immediately drawn to this beautiful problem. At the time I knew few tools in extremal combinatorics and graph theory, so I was mostly playing with the problem with my bare hands. Learning from Richard Stanley's classes and textbooks got me to be quite comfortable with bijection arguments, which came in handy. I played with a lot of small examples and eventually found a way to injectively map independent sets of $G \sqcup G$ (two copies of some arbitrary graph $G$ ) into those of $G \times K_{2}$ (the bipartite double cover of $G$ ). This was exactly what was needed to extend Kahn's result from bipartite graphs to general graphs.

It took me a while to internalize Kahn's beautiful entropy argument for the bipartite case. When I first read it, I could follow Kahn's proof line by line but did not really understood why the argument worked. Later I found (in a joint paper with Eyal Lubetzky) ${ }^{32}$ a new proof of Kahn's result using Hölder's inequal- ity, without using any entropy - also cleanly reproving a generalization by Galvin and Tetali ${ }^{33}$ to graph homomorphisms. We were actually working on a seemingly unrelated problem about large deviations in random graphs, but it turned out that the same Hölder's inequality trick worked in both. Finally, it was only when I taught a class on the probabilistic method, covering the entropy method, that I revisited Kahn's proof and finally understood it.

More recently, with Ashwin Sah, Mehtaab Sawhney, and David Stoner (all undergraduates at the time), we proved two long soughtafter extensions of the independent set result, namely to irregular graphs ${ }^{34}$ and to colorings ${ }^{35}$. Our technique is heavily analytic (for example, lots of applications of Hölder's inequality) rather than entropic. I would be interested to see simpler proofs, perhaps using the entropy method again.

Mansour: Would you tell us about your thought process for the proof of one of your favourite results? How did you become interested in that problem? How long did it take you to figure out a proof? Did you have a "eureka moment"?

Zhao: For the independent set in regular graphs result I discussed above, I fondly remember thinking about the problem for several weeks while sitting in an empty reading room in a Duluth library. It was a distractionfree bliss. I was elated when I finally discovered the proof. This was my first real "eureka moment," and it remains one of my happiest mathematical memories.

Mansour: Is there a specific problem you have been working on for many years? What progress have you made?

Zhao: I really like the sphere packing problem in high dimensions: what is the maximum density of a packing of unit balls in $\mathbb{R}^{n}$, asymptotically for large $n$ ? Henry Cohn is a leading expert on sphere packing, and he infected me with his interest on the subject when I was a

\footnotetext{
${ }^{30}$ Y. Zhao, The number of independent sets in a regular graph, Combin. Probab. Comput. 19 (2010), 315-320.

${ }^{31} \mathrm{~N}$. Alon, Independent sets in regular graphs and sum-free subsets of finite groups, Israel J. Math. 73 (1991), $247-256$.

${ }^{32}$ E. Lubetzky and Y. Zhao, On replica symmetry of large deviations in random graphs, Random Structures Algorithms 47 (2015), 109-146.

${ }^{33}$ D. Galvin and P. Tetali, On weighted graph homomorphisms, Graphs, morphisms and statistical physics, DIMACS Ser. Discrete Math. Theoret. Comput. Sci., vol. 63, Amer. Math. Soc., Providence, RI, 2004, pp. 97-104.

${ }^{34}$ A. Sah, M. Sawhney, D. Stoner and Y. Zhao, The number of independent sets in an irregular graph, J. Combin. Theory Ser. B 138 (2019), 172-195.

${ }^{35}$ A. Sah, M. Sawhney, D. Stoner and Y. Zhao, A reverse Sidorenko inequality, Invent. Math. 221 (2020), 665-711.

${ }^{36}$ G. A. Kabatiansky and V. I. Levenshtein, Bounds for packings on the sphere and in space, Problemy Peredači Informacii 14
} 
student.

In the 70's, Kabatiansky and Levenshtein ${ }^{36}$ proved an upper bound of $2^{-(0.599 \cdots+o(1)) n}$, and this is essentially the best known in high dimensions. Cohn and $\mathrm{I}^{37}$ managed to improve this bound by an embarrassingly tiny amount - a constant factor in front of the expression (not in the exponent).

As for the lower bound, an easy greedy argument shows that one can pack with density at least $2^{-n}$. This bound was subsequently improved to $c n 2^{-n}$. Recently, Venkatesh ${ }^{38}$ proved a lower bound of the form $c n(\log \log n) 2^{-n}$ for a sparse infinite sequence of $n$ 's. This is the best of our knowledge, but it is almost certainly not the end of the story.

Every expert I talk to believes that lattice packings cannot be significantly denser than $2^{-n}$ (maybe even $<n^{2} 2^{-n}$ ). But, well, I suspect that there are exponentially denser lattices, but I have nothing concrete at the moment, so maybe I will just leave it at that.

Mansour: Professor Yufei Zhao, I would like to thank you for this very interesting interview on behalf of the journal Enumerative Combinatorics and Applications. 